\title{
A Fuzzy Control Design for the Trajectory Tracking of Autonomous Mobile Robot
}

\author{
Yung-Hsiang Chen ${ }^{1}$, Chan-Hong Chao ${ }^{2}$, Chun-Fa Cheng ${ }^{3}$ and Chiou-Jye Huang ${ }^{4 *}$ \\ ${ }^{1}$ Department of Mechanical Engineering, National Pingtung University of Science and Technology, Taiwan \\ ${ }^{2}$ Department of Electrical Engineering, Kun-Shan University, Taiwan
}

${ }^{3}$ Graduate Institute of Hakka Cultural Industry, National Pingtung University of Science and Technology, Taiwan

${ }^{4}$ School of Electrical Engineering and Automation, Jiangxi University of Science and Technology, China

*Corresponding author: Chiou-Jye Huang, School of Electrical Engineering and Automation, Jiangxi University of Science and Technology, China

\begin{abstract}
In this study, a fuzzy control design for the trajectory tracking of autonomous mobile robot (AMR) will be presented. This proposed fuzzy control approach can be applied to track the desired trajectory of AMR. The main objective and contribution are to indicate a fuzzy control law which can drive the AMR to track a desired trajectory for the nonlinear trajectory tracking control of AMR. Generally, it is a difficult task to derive the solution from this nonlinear trajectory tracking problem, so we will try to deal with this trajectory tracking problem by fuzzy control design approach. Finally, a testing scenario of S-type reference trajectory tracking is verified the tracking performance of AMR.
\end{abstract}

Keywords: Fuzzy control design; Trajectory tracking; Autonomous mobile robot (AMR)

\section{Introduction}

More and more autonomous mobile robots (AMRs) are extensively applied in various fields which include industry and service in the past decades. According to above reasons, the precise manipulations of AMR are very important and key in the trajectory tracking topic. A lot of existing research [1-10] about trajectory tracking problem are discussed. But these research are too complicated and not easy to be implemented. Based on this reason, we will propose a fuzzy control design for the trajectory tracking of AMR in this paper.

\section{The Mathematical Model of AMR}

The mathematical model and tracking error dynamic equation of AMR will be presented in this section. This approach purpose is to construct a fuzzy control method for the tracking error to close to zero quickly and let the AMR traces the desired trajectory successfully. Base on this mathematical model and the geometry relationship with the AMR, a nonlinear tracking error and tracking error dynamic equation for the controlled AMR can be described as following equations (1) and (2), respectively [11].

The definition of the tracking error between the desired tracking trajectory and AMR can be described as below equation.

$$
e=\left[\begin{array}{l}
\dot{\hat{r}} \\
\hat{r}
\end{array}\right]=\left[\begin{array}{l}
\dot{r}-\dot{r}_{r} \\
r-r_{r}
\end{array}\right]
$$

where: $r=\left[\begin{array}{lll}x_{c} & y_{c} & \theta\end{array}\right]^{T}$ and $r_{r}=\left[\begin{array}{lll}x & y & \theta_{d}\end{array}\right]^{T}$

and the tracking error dynamic equation is given as following equation. 
$\dot{e}=\left[\begin{array}{cc}-H^{-1}(r) C(r, \dot{r}) & O_{3 \times 3} \\ I_{3 \times 3} & O_{3 \times 3}\end{array}\right] e+\left[\begin{array}{c}-\ddot{r}_{r}-H^{-1}(r) C(r, \dot{r}) \dot{r}_{r} \\ O_{3 \times 3}\end{array}\right]+\left[\begin{array}{c}H^{-1}(r) T(r) \tau \\ O_{3 \times 3}\end{array}\right](2)$

where: $H(r) \in R^{3 \times 3}$ is a symmetric positive definite inertia matrix and $C(r, \dot{r}) \in R^{3 \times 3}$ is the centripetal and Coriolis matrix. $T(r) \in R^{3 \times 2}$ is the input transformation matrix and $\tau \in R^{2 \times 1}$ is the applied to the torque vector.

\section{Autonomous Mobile Robot Fuzzy Controller Design}

\section{Fuzzifier:}

The input and output variables of a fuzzy system are the linguistic variables and will be taken as fuzzification by membership function in this section. The input and output linguistic variables of fuzzy logic-based include:
1. Tracking error in $\mathrm{X}$ axis $\mathrm{e}_{\mathrm{x}}$.

2. Tracking error in $\mathrm{Y}$ axis $\mathrm{e}_{\mathrm{y}}$.

3. Rotation tracking error angle $\theta$.

4. The output variable is control torques $\tau$.

The linguistic values treat by these variables are expressed by linguistic sets. Each of linguistic variables is assumed to take five linguistic sets and are defined as large negative (LN), small negative (SN), zero (ZE), small positive (SP) and large positive (LP). The linguistic sets are described by their membership functions. From Figures 1-4, the membership functions are described for X-axis tracking error $\mathrm{e}_{\mathrm{x}}$ is $[-1,1]$ meter, $\mathrm{Y}$-axis tracking error $\mathrm{e}_{\mathrm{y}}$ is $[-4,4]$ meter, angle tracking error $\theta$ is $[-30,30]$ degree and control torque output $\tau$ is $[-2,2] \mathrm{Nm}$, respectively. To simplify the computation in the actual operation, triangular membership functions are suggested.

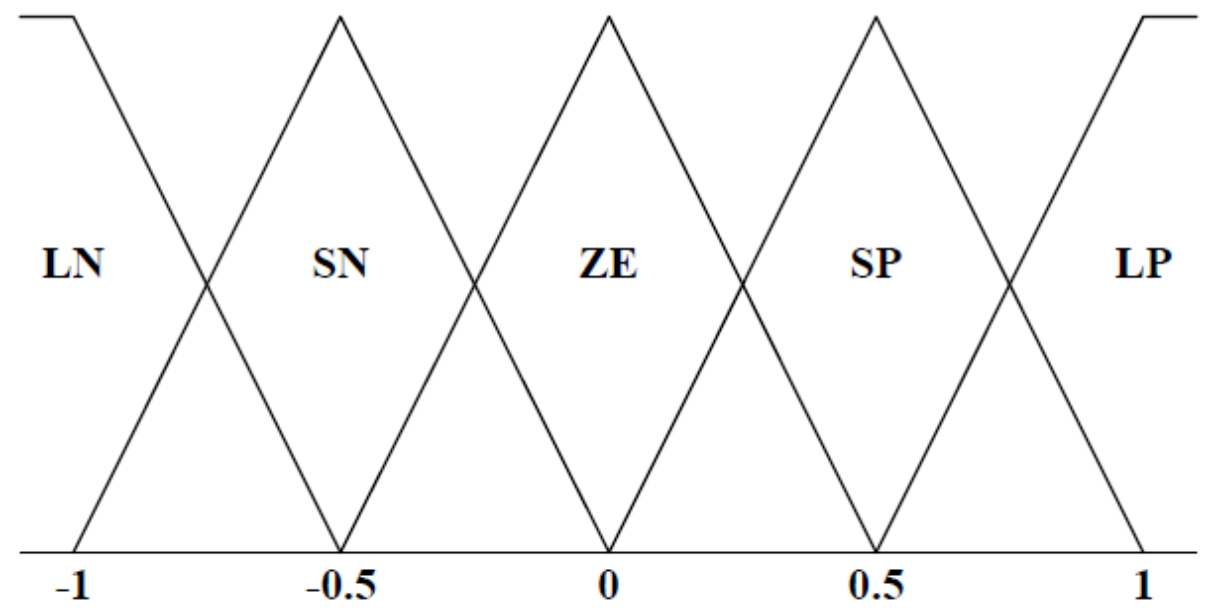

Figure 1: The membership function of $\mathrm{X}$-axis tracking error

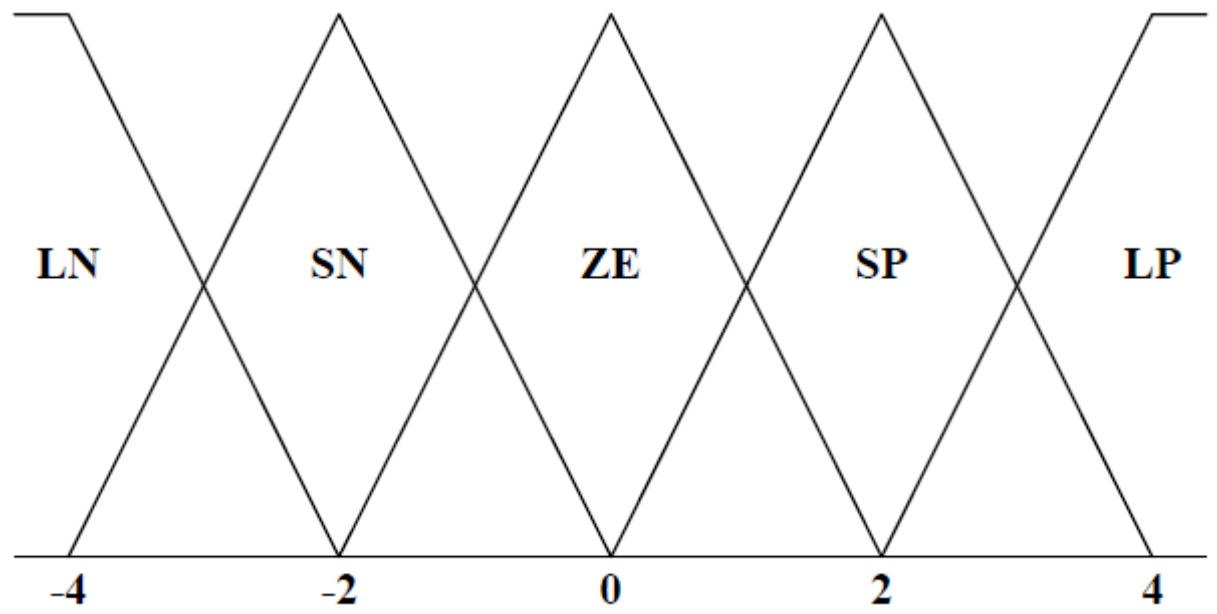

Figure 2: The membership function of Y-axis tracking error. 


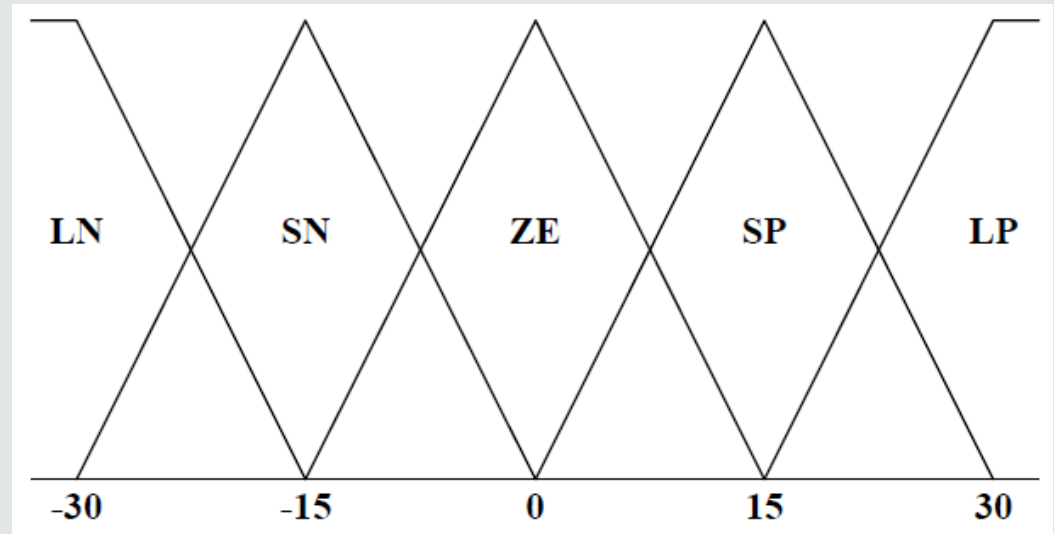

Figure 3: The membership function of angle tracking error.

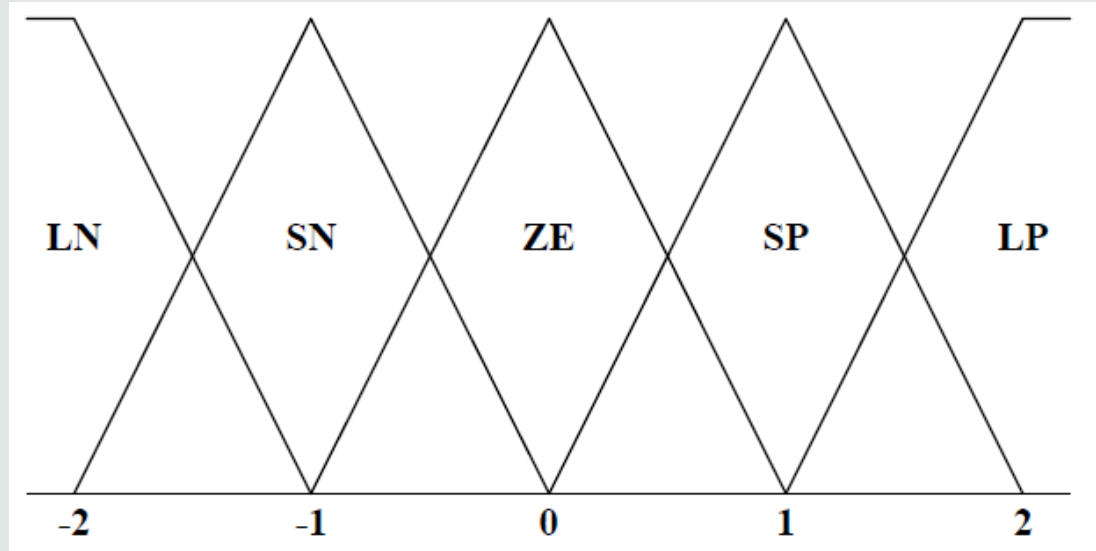

Figure 4: The membership function of control torque output.

\section{Defuzzifier:}

The outputs of the linguistic rules are fuzzy sets, but the control command must be a single crisp value. Therefore, the outputs of linguistic rules must be defuzzified before inputting to the AMR system. The fuzzy results will be converted into a single crisp value with respect to a fuzzy set and calculated by using the center-ofgravity (COG) defuzzification process.

\section{Simulation Result}

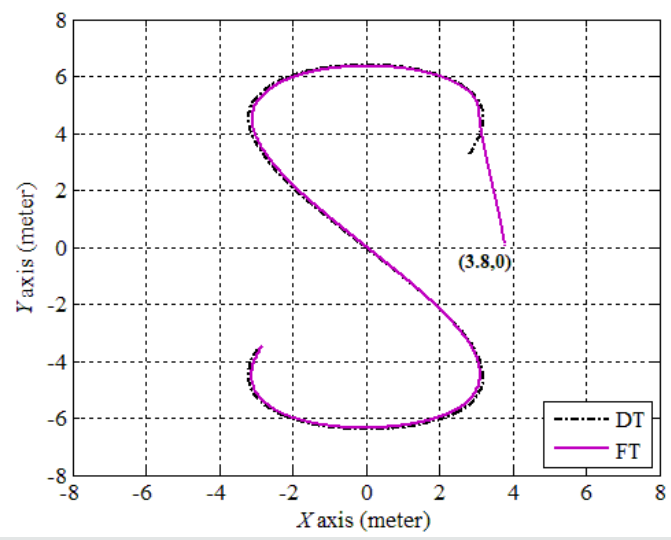

Figure 5: S-type trajectory tracking result from $(x=3.8$ meter, $y=0$ meter). (Desired Trajectory (DT): back line and (Fuzzy Trajectory (FT): purple line) 
A desired S-type reference trajectory tracking will be verified by this proposed fuzzy control design in this section. From simulation results, the verification result of the proposed method of a desired S-type reference trajectory tracking with a radius of 3.8 meters shows in Figure 5. As the significant results of the tracking error of the proposed method to the desired S-type trajectory reveal an outstanding performance from Figures 6-10. According to the obvious simulation results, this proposed method can guide the controlled AMR to track the S-type reference trajectory precisely and quickly.

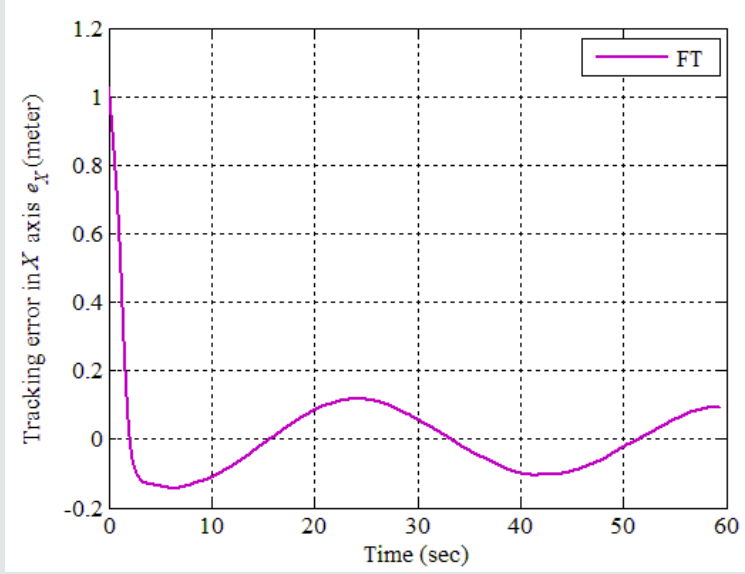

Figure 6: The tracking error result of $X$ axis from $(x=3.8$ meter, $y=0$ meter $)$.

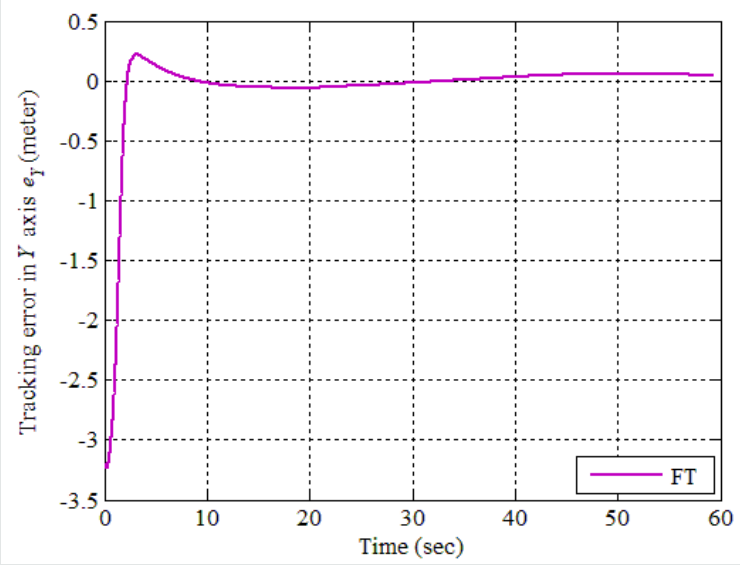

Figure 7: The tracking error result of $Y$ axis from $(x=3.8$ meter, $y=0$ meter).

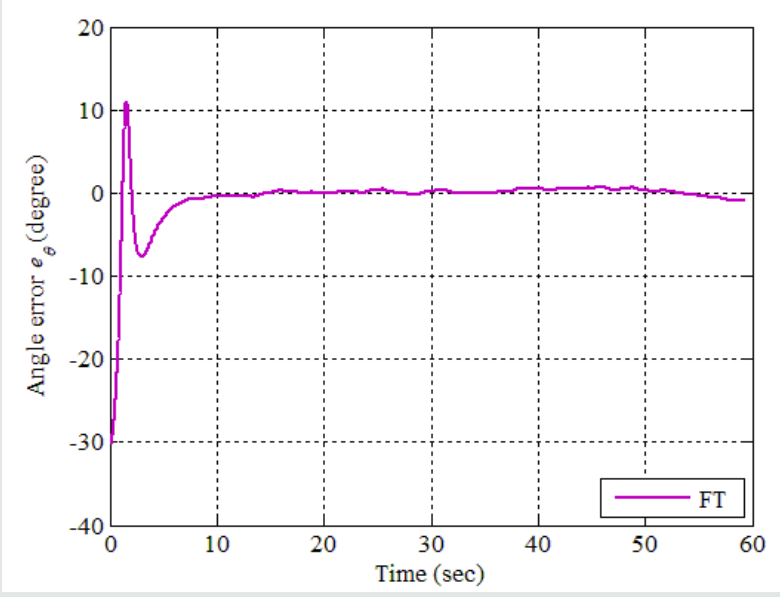

Figure 8: The tracking error result of angle from $(x=3.8$ meter, $y=0$ meter). 


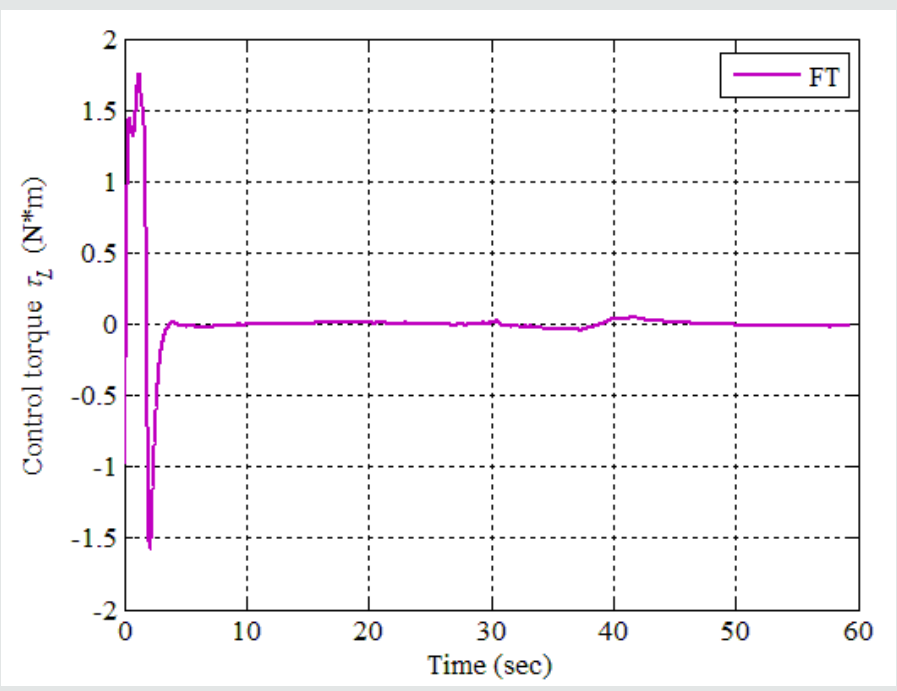

Figure 9: The output result of left torque from ( $x=3.8$ meter, $y=0$ meter).

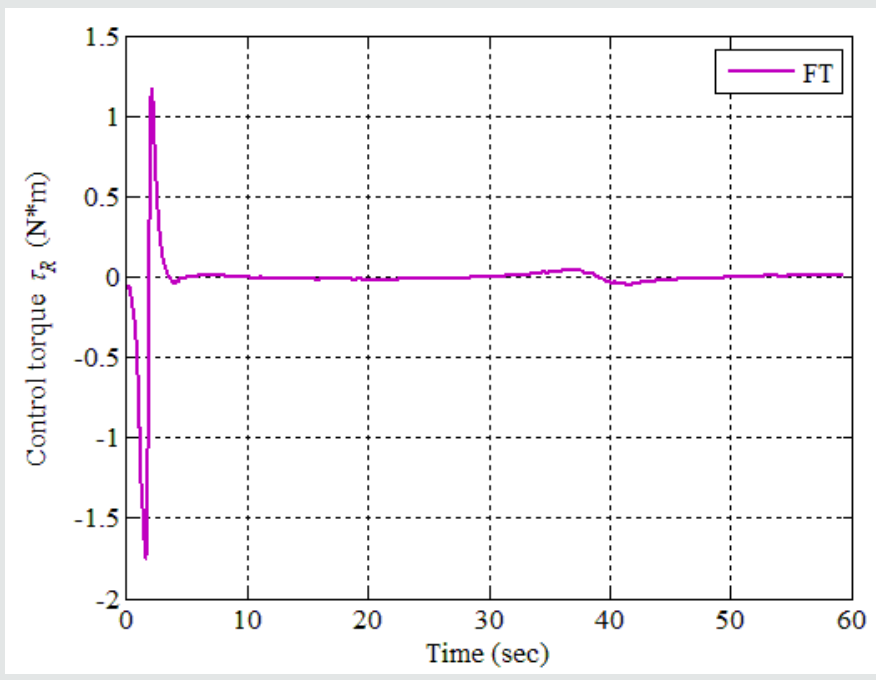

Figure 10: The output result of right torque from $(x=3.8$ meter, $y=0$ meter).

\section{Conclusion}

In this manuscript, a proposed fuzzy control design approach is successfully developed for improving the S-type reference trajectory tracking capability of nonlinear AMR system. As the simulation results, the proposed fuzzy control design approach demonstrates a satisfactory performance for tracking the desired trajectory in S-type reference trajectory.

\section{References}

1. Chen YH (2020) A Novel Fuzzy Trajectory Tracking Control Design for Wheeled Mobile Robot. Advances in Robotics \& Mechanical Engineering 2(3): 142-144.

2. Abdelwahab M, Parque V (2020) Trajectory Tracking of Autonomous Mobile Robots Using Z-Number Based Fuzzy Logic. IEEE Access 8(8): 18426-18441.
3. Hacene N, Mendil B (2019) Fuzzy Behavior-based Control of Three Autonomous Omnidirectional Mobile Robot. International Journal of Automation and Computing 16(2): 163-185.

4. Chen YY, Chen YH, Huang CY (2018) Autonomous mobile robot design with robustness properties. Advances in Mechanical Engineering 10(1): $1-11$.

5. Tiep D K, Lee K, Im DY, Kwak B, Ryoo YJ (2018) Design of Fuzzy-PID Controller for Path Tracking of Mobile Robot with Differential Drive. International Journal of Fuzzy Logic and Intelligent Systems 18(32): 220-228.

6. Wu TF (2018) Tracking Control of Autonomous Mobile Robots Using Fuzzy CMAC Neural Networks. Journal of Internet Technology 19(6): 1853-1869.

7. Chen YH, Li TH, Chen YY (2013) A Novel Nonlinear Control Law with Trajectory Tracking Capability for Nonholonomic Mobile Robots: Closed- Form Solution Design. Applied Mathematics and Information Sciences 7(2): 749-754. 
8. Chen CY, Li TH, Yeh YC (2009) EP-Based Kinematic Control and Adaptive Fuzzy Sliding-Mode Dynamic Control for Autonomous Mobile Robots. Information Sciences 179(1): 180-195.

9. Tamoghna D, Kar I, Chaudhury S (2006) Simple Neuron-based Adaptive Controller for a Nonholonomic Mobile Robot Including Actuator Dynamics. Neurocomputing 69(16): 2140-2151.

10. Oriolo G, Luca A, Vendittelli M (2002) WMR Control via Dynamic Feedback Linearization: Design, Implementation and Experimental Validation. IEEE Transaction Control System Technology 6(10): 835852.
11. Chen YH, Li TH, Chen YY (2013) A Practical Trajectory Tracking Approach for Autonomous Mobile Robots: Nonlinear Adaptive H2 Design. The Transactions of the Canadian Society for Mechanical Engineering 37(3): 385-394.

\section{(C) This work is licensed under Creative}

To Submit Your Article Click Here: Submit Article

DOI: $10.32474 / A R M E .2020 .02 .000148$

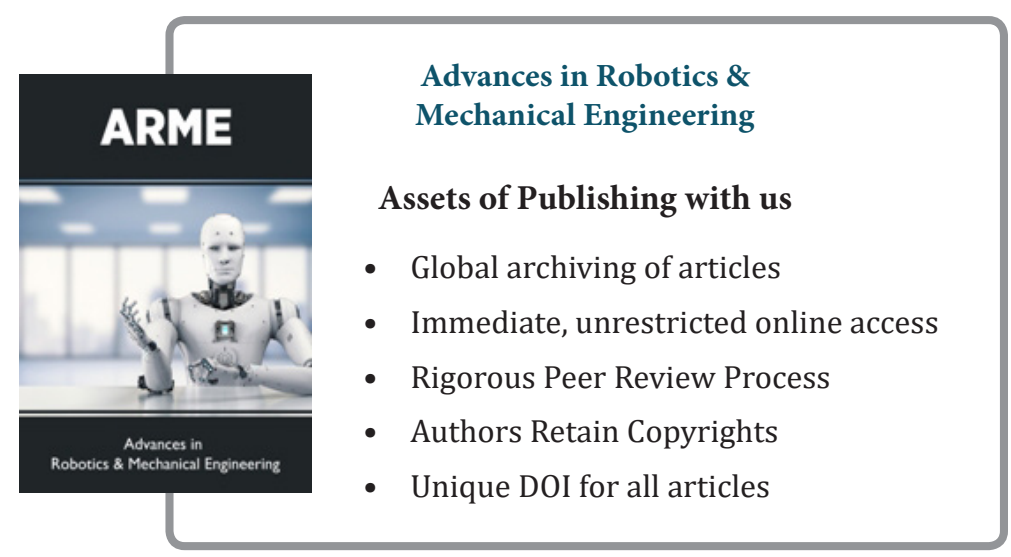

